\title{
Designing Tactile Vocabularies for Human-Computer Interaction
}

\author{
Author: Victor Adriel de Jesus Oliveira, Adviser: Prof. Dr. Anderson Maciel \\ ${ }^{1}$ Instituto de Informática - Universidade Federal do Rio Grande do Sul (UFRGS) \\ Caixa Postal 15.064 - 91.501-970 - Porto Alegre - RS - Brazil \\ \{vajoliveira, amaciel\}@inf.ufrgs.br
}

\begin{abstract}
This paper summarizes our study of tactile languages in humancomputer interaction. We intended to analyze how the choices made during the design process of tactile vocabularies would affect the user performance on an interactive task. Therefore, we have developed and tested different sets of tactile signals for aid navigation in virtual environments. It leaded us to fashion a novel approach for vibrotactile prefixation. Through this experimental-driven study, we attempted to effects of multisensory stimulation, perception, learning and interpretation of tactile sequences, and masking caused by multiple vibrations in a same locus. The presented results should be useful for other designers to produce usable and expressive tactile interfaces.
\end{abstract}

\section{Introduction}

The greatest challenge in the area of tactile communication was well described by Sherrick [Sherrick 1991] as the discovery of a set of tactile patterns that, as speech sounds or letters, are clearly discriminated, rapidly processed and easily learned. In most of the mechanisms designed for tactile communication, the set of tactile patterns follows an approach known as "tap-on-shoulders" [Erp et al. 2005]. Although tactile patterns with such characteristic use to be easy to understand, they are also limited and provide a relatively small amount of information to the user.

We aimed to discover how to increase the expressiveness of a tactile language, keeping it as easy as possible to learn and to understand. Therefore, we explored design approaches for tactile languages and proposed a novel approach for prefixation. Different kinds of information can be expressed by the use of various parameters as, for example, variations in intensity and frequency of vibrators in a vibrotactile display. The meaning of each parameter is assigned arbitrarily. The addition of a small set of arbitrary elements may increase the complexity of the language, but also allows the transmission of a greater amount of information. The present work focuses on the study of how to use and combine such elements.

The proposed approach for prefixation was used to design vibrotactile patterns for aid navigation tasks, and it was tested in virtual environments. The messages transmitted to support navigation in virtual environments should also be used for navigation in physical environments. Therefore, it is expected that the same vibrotactile language could be applied with few adjustments to several applications, such as gaming, robot-teleoperation systems, pedestrian orientation and so on. The development of tactile vocabularies and the research on vibrotactile prefixation was conducted through an iterative and incremental process. So, we present our finds from the perspective of a cyclic set of steps of (re)design, prototyping, user experiments and analysis of different tactile vocabulary designs. 


\section{Related Work}

Formerly, many tactile applications aimed to replace the senses, thinking about tactile devices as a good alternative for deaf and blind people. However, several more recent applications focus on complementing senses instead [MacLean and Hayward 2008]. The haptic feedback may be not as suitable as visual or auditory stimuli for some tasks. However, it is appropriate as complementary modality when other senses are already loaded, implying multitasking and disruptions. Therefore, our work focused on the assessment of multisensory interfaces for sighted subjects.

Design an unfamiliar modality has challenges, though. People are not habituated to processing haptic representations of abstractions, and there is no predefined tactile vocabulary to describe such representations. Thus, inspired by the works about tactile icons [Brewster and Brown 2004], tactile vocabularies [MacLean and Hayward 2008, Israr and Poupyrev 2011] and methods for the design of tactons [Riddle and Chapman 2012, Chan et al. 2008], we created and assessed tactile vocabularies aiming its usability and expressivity.

\section{Experimental-Guided Study}

We emphasized the use of a prefixation approach for the construction of tactons. That way it could be possible to reuse the same patterns to convey different meanings, just by concatenating them with prefixal signals. So, based on Braille, we have defined the concept of Modifier Tactile Pattern as a tactile signal that modifies the interpretation of the remaining signals of a Tacton or tactile sequence. By reusing patterns, we could get tactile vocabularies made by fewer signals to recall. Thus, the use of modifiers could enhance the tactile vocabulary expressiveness keeping it easy to learn.

To evaluate such characteristic we have designed several tactile vocabularies and performed user tests in virtual environments. Therefore, our research can be summarized in two parts: our approach for tactile prefixation and the cycles of design, redesign, and assessment of tactile vocabularies.

\subsection{Novel Approach for Construction of Tactile Messages}

To reduce the number of chunks of a tactile language as we increase its expressiveness, we have found inspiration from morphology and formalized the concept of Modifier Tactile Pattern for vibrotactile displays. Arbitrary variations may result in vocabularies that are difficult to memorize and process. The use of prefixation with modifiers could enhance the tactile vocabulary expressiveness keeping it easier to learn due the number of signals to recall.

The concept of Modifier Tactile Pattern is subject to some of the same rules that govern the use of modifier signs in Braille. However, the Modifier works differently for haptizations. Vibrotactile cues are dynamic and are composed, of several parameters. In Braille, the modifier affects one or more patterns in sequence (see Figure 1) while the Modifier can affect patterns or vibrotactile parameters sequentially or simultaneously. In our work, we present not only the concept of Modifier Tactile Pattern but also the means to design tactile icons with modifiers. We also present the assessment of different modifierbased vocabularies. 


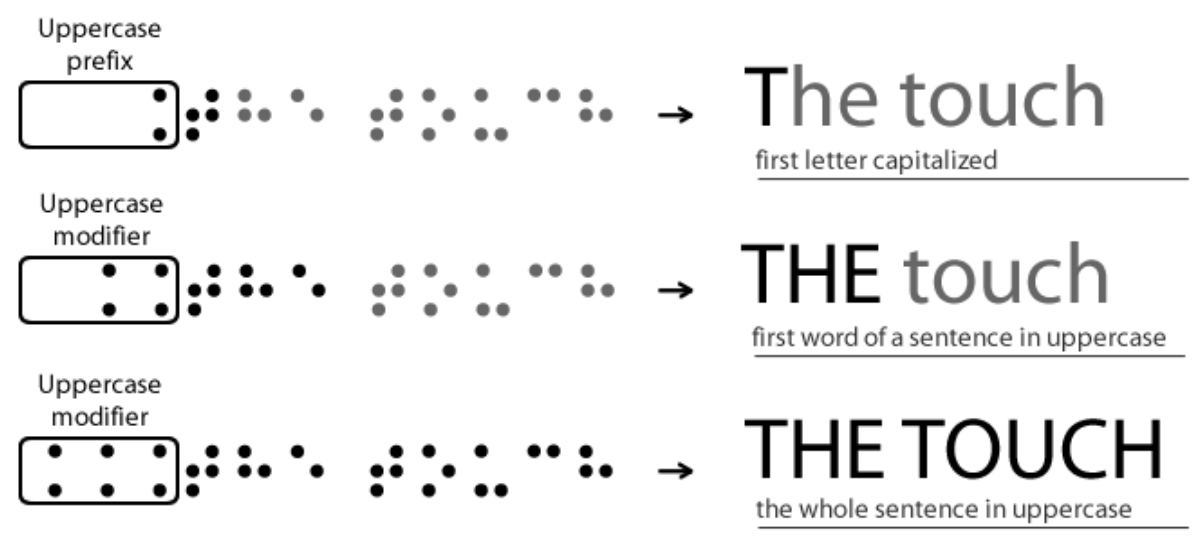

Figure 1. Uppercase modifier in Braille formed by concatenating the uppercase prefix. Such mechanism inspired the concept of Modifier Tactile Pattern, which works differently for tactile vocabularies.

\subsection{Assessment of Tactile Vocabularies}

From now on, we summarize the design and assessment of modifier-based vocabularies which led us to a final usable vocabulary for navigation.

\subsubsection{Initial Vocabulary for Tactile Navigation}

As a means to ascertain the feasibility of designing tactile interfaces, we designed and tested this primary vocabulary to characterize elements of a path, such as obstacles and routes, to aid navigation. The tactile display was built for delivering tactile cues on the palm of the user's hand (see Figure 2). And, although the Modifier concept was not formalized yet, it was applied experimentally in this initial design. Either perception of the tactile cues and interpretation of the tactile icons were tested in user experiments. The experiments have shown the usefulness of the created tactile vocabulary while aid for orientation and navigation tasks. Even without acquaintance with this kind of interaction, all participants performed the task successfully, reaching the destination with the support of the tactile display. The test results inspired new strategies to optimize the displaying of the tactile icons on the hand and helped us to shape the concept of Tactile Modifier Pattern.

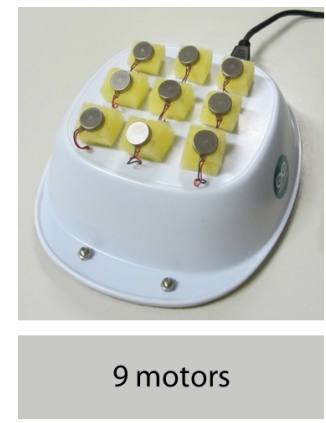

(a)

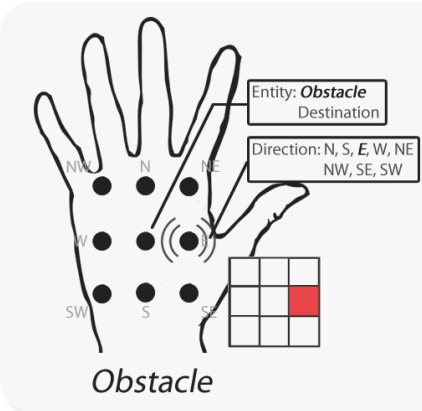

(b)

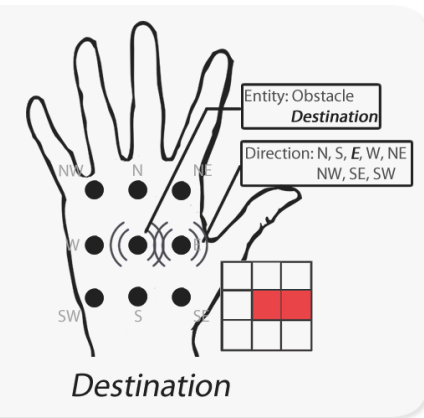

Figure 2. (a) Final prototype of the hand-based vibrotactile display. And, (b) example of Tactons. 


\subsubsection{Enhancement of the Tactile Language}

In a second iteration, we created an enlarged version of the tactile language for navigation. This version was intended to be more expressive. So, the message set was increased to transmit more information during the navigation task. The tactile language was also intended to be suitable for navigation in virtual environments and also physical environments. Thus, the prototype of the tactile display was redesigned as a belt to become wearable and deliver information from an egocentric perspective.

We designed three vocabularies and conducted user studies to assess the differences between tactile vocabularies made by a prefixation and a non-prefixation approach, and also the characteristics of prefixed messages by superposition and juxtaposition (see Figure 3). Each vocabulary follows a different approach: a conventional one, based only on metaphors; a modifier-based vocabulary with patterns made by a juxtaposition of tactons in sequence; and another modifier-based vocabulary with patterns made by superposition. Analyzing the users performance we observed how it was influenced by tactile sequences and a larger number of stimuli at a same time. Even the difference between the performances of the three groups was not significant, the modifier-based vocabularies afforded the best results.

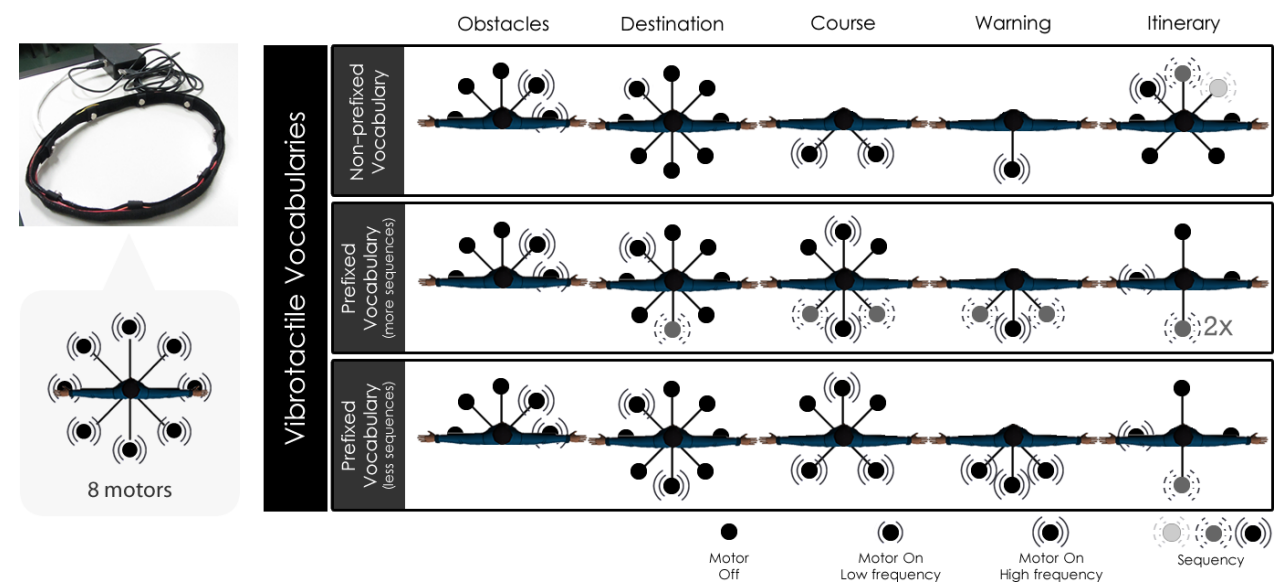

Figure 3. Comparison between vocabularies. A top view of a humanoid with vibrators positioned at equidistant locations around its waist represents each tactile pattern.

\subsubsection{Redesign and Application Trial}

In this last iteration, we performed a perceptual adjustment of our tactile language and implemented it for the navigation in a virtual underground mine (see Figure 4). Underground mines have large areas that are not enlightened and many times the miners count only with the light from their mining helmet. Due the use of explosives, underground slips, and the mineral exploration, the mine galleries can contain smoke and powder fumes that can difficult the vision. In the underground environment, the noise is also frequent. Therefore, the tactile feedback seems to be suitable to aid the mine workers in their tasks. To test this, we designed a virtual representation of a mine and simulated a risk situation in which the user should navigate in the mine to reach a rescue chamber. In a real emergency situation, 
the life of the mine worker relies on the right perception of the tactile icons delivered by the tactile display. Therefore, the usability of the haptic interface is crucial. In this final vocabulary, the signals were chosen focusing on the user and the task, which allowed us to improve the usability of our tactile application significantly.

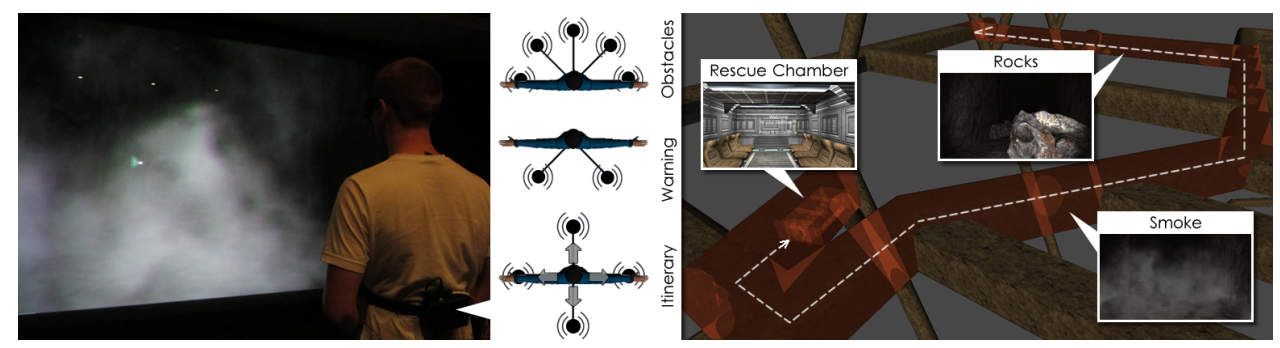

Figure 4. Redesigned vocabulary for underground navigation. (a) shows a user performing the navigation task and (b) shows the 3D virtual representation of an underground mine.

\section{Publications}

Our contributions are related to the fields of Human-Computer Interaction, Virtual Reality, and Haptics. Our first findings, with a vibrotactile display for the back of the hand, were published as Work in Progress at the National Conference on Graphics, Patterns and Images - SIBGRAPI (B1) [Oliveira and Maciel 2012]. After that, we redesigned the display for delivery patterns on the palm of the user's hand, performed experiments, and formalized and exemplified the concept of Tactile Modifier Pattern, our major contribution [Oliveira and Maciel 2014b]. It was published in the international conference EUROHAPTICS (B1), one of the most important conferences on Haptics. Our assessment of different vocabularies for navigation in virtual environments using a vibrotactile display as a belt was also published in the EUROHAPTICS Conference [Oliveira and Maciel 2014a] and as a poster at the IEEE Symposium on 3D User Interfaces (B2) [Oliveira and Maciel 2014c]. Our final vocabulary for navigation in underground mines was the Best Application Paper in the National Symposium on Virtual and Augmented Reality - SVR (B4) [Oliveira et al. 2014] and their results helped us to design a vibrotactile display for intercommunication, presented as a poster at the International IEEE Virtual Reality Conference (A2) [Oliveira et al. 2015].

\section{Conclusions}

We studied the prefixation as a way to increase the expressiveness of a tactile language, and we proposed a novel approach for vibrotactile prefixation. This approach was studied in an iterative and incremental process and presented as cycles of design-analysis-redesign of tactile vocabularies for navigation in virtual environments. The result of this study is a compendium that contains a large review of the research on vibrotactile communication and the analysis of different tactile vocabularies and displays for navigation. More than eighty people have participated in the user experiments. We prototyped tactile displays for different body sites covering the glabrous skin (hand) and the hairy skin (waist). We also addressed the effects of multisensory stimuli and sensory substitution in our experiments through the navigation on completely dark scenarios and enlightened ones. And finally, we have formalized the concept of Modifier Tactile Pattern for vibrotactile displays. 
The prototyping of haptic devices is the focus of many other works. However, we chose to concentrate on an experimental-guided study to explore the potential of vibrotactile languages. Our results can contribute to the construction of other haptic interfaces for navigation, but there is a lot of work to do in the study of the effects of workload and multisensory stimulation. We expect that our vibrotactile language can be applied with few adjustments to several other applications, such as gaming, robot-teleoperation systems, pedestrian orientation and so on. We have designed and tested a dozen families of tactile icons; those results can shorten the way to the development of a standard grammar for tactile navigation. With the ascension of wearable devices with small contact surfaces, such as smart watches, smart glasses, and portable HMDs, the use of prefixes and modifiers can increase the capability of Tacton construction.

\section{References}

Brewster, S. and Brown, L. M. (2004). Tactons: structured tactile messages for non-visual information display. AUIC '04, pages 15-23. Australian Computer Society, Inc.

Chan, A., MacLean, K., and McGrenere, J. (2008). Designing haptic icons to support collaborative turn-taking. International Journal of Human-Computer Studies, 66(5):333355.

Erp, J. B. V., Veen, H. A. V., Jansen, C., and Dobbins, T. (2005). Waypoint navigation with a vibrotactile waist belt. ACM Transactions on Applied Perception, 2(2):106-117.

Israr, A. and Poupyrev, I. (2011). Tactile brush: drawing on skin with a tactile grid display. In Proceedings of the SIGCHI Conference on Human Factors in Computing Systems, CHI '11, pages 2019-2028, New York, NY, USA. ACM.

MacLean, K. E. and Hayward, V. (2008). Do it yourself haptics: Part ii. Robotics \& Automation Magazine, IEEE, 15(1):104-119.

Oliveira, V. A. d. J. and Maciel, A. (2012). Using vibrotactile communication to assist in orientation and locomotion. In D. Menotti, A. C. N. J., editor, Workshops of SIBGRAPI (2012), pages 47-48, Ouro Preto, MG, Brazil.

Oliveira, V. A. d. J. and Maciel, A. (2014a). Assessment of tactile languages as navigation aid in 3d environments. In EUROHAPTICS, 2014.

Oliveira, V. A. d. J. and Maciel, A. (2014b). Introducing the modifier tactile pattern for vibrotactile communication. In EUROHAPTICS, 2014.

Oliveira, V. A. d. J. and Maciel, A. (2014c). Poster: Applying tactile languages for 3d navigation. In 3D User Interfaces (3DUI), 2014 IEEE Symposium on, pages 177-178.

Oliveira, V. A. d. J., Marques, E., Peroni, R. L., and Maciel, A. (2014). Tactile interface for navigation in underground mines. In SVR, 2014.

Oliveira, V. A. d. J., Sarmiento, W., Maciel, A., Nedel, L., and Collazos, C. (2015). Does vibrotactile intercommunication increase collaboration? In IEEE VR, 2015.

Riddle, D. L. and Chapman, R. J. (2012). Tactile language design. In Proceedings of the Human Factors and Ergonomics Society Annual Meeting, pages 478-482.

Sherrick, C. (1991). Vibrotactile pattern perception: Some findings and applications. The psychology of touch, pages 189-217. 